\title{
EDUCACIÓN INCLUSIVA Y TIC: SISTEMAS DE BARRIDO OCULAR PARA ALUMNADO CON PARÁLISIS CEREBRAL EN EDUCACIÓN PRIMARIA
}

\section{INCLUSIVE EDUCATION AND ICT: EYE-TRACKING SYSTEMS FOR STUDENTS WITH CEREBRAL PALSY IN PRIMARY EDUCATION}

\author{
Rosa María Espada-Chavarría \\ rosa.espada@urjc.es \\ Ricardo Moreno-Rodríguez \\ ricardo.moreno@uric.es \\ Facultad de Ciencias Jurídicas y Sociales. \\ Universidad Rey Juan Carlos (España) \\ Mariano Morán-Montalvo \\ mariano.moran@educa.madrid.org
}

CEIP Pinar de San José. Madrid (España).

Recibido: $12 / 10 / 2020$

Aceptado: 09/12/2020

\begin{abstract}
Resumen:
El uso de las Tecnologías de la Información y Comunicación (TIC) como herramienta de acceso al currículo, supone uno de los grandes avances para alcanzar una educación más inclusiva. Se presenta el estudio de uno de los dos casos en España, que explora la implementación y el proceso llevado a cabo en un centro educativo para hacer efectiva la inclusión educativa de una alumna de 10 curso de Educación Primaria que presenta parálisis cerebral con predominio espástico, lo que supone una dificultad en el control muscular, siendo solo este controlado de manera ocular, por lo que se han buscado alternativas para su acceso al currículo a través del uso de dispositivos de barrido ocular, sistemas aumentativos de comunicación y TIC. Los resultados avanzan la idoneidad del proceso de inclusión que supone la presencia, participación y aprendizaje en igualdad de oportunidades y los óptimos resultados de aprendizaje utilizando en exclusiva adaptaciones de acceso al currículo oficial.
\end{abstract}

Palabras clave: Barrido ocular, comunicación aumentativa, educación inclusiva, inclusión, parálisis cerebral, TIC.

\footnotetext{
Abstract:

Information and Communication Technologies (ICT) are an essential tool for achieving inclusive education. We present a research that studies one of the two cases in Spain of a 1st grade primary school student with cerebral palsy with spasticity, who only has ocular muscle control. Alternatives have been sought to access the curriculum on an equal opportunity basis, using
} 
eye-tracking devices, augmentative communication systems, and ICT. The results advance the adequate inclusion process that demonstrates presence, participation and learning among equals. In addition, good results in learning qualifications are guaranteed. To access the curriculum, only access adaptations have been made, not significant adaptations.

Key words: Eye-tracking, augmentative communication, inclusive education, inclusion, cerebral palsy, ICT

\section{Introducción}

En los últimos años, la difusión y extensión de la idea de mejorar la calidad de la educación para todos, tal y como se establece en la Ley Orgánica 8/2013, de 9 de diciembre, para la Mejora de la Calidad Educativa (LOMCE), junto con el ya conocido Objetivo de desarrollo sostenible no 4, de educación de calidad, que plantea "Garantizar una educación inclusiva y equitativa de calidad y promover oportunidades de aprendizaje permanente para todos" lleva al reto de conseguir una escuela inclusiva que elimine los procesos de exclusión en educación y promueva una atención equitativa y de calidad a todo el alumnado dentro de entornos educativos generales. Este hecho, supone repensar que entendemos por educación inclusiva y cómo la estamos concretando en la práctica real. Por tanto, este punto de partida justifica la importancia de compartir casos como el que nos ocupa, que reflejan la necesidad de emprender proyectos de cambio y mejora, relacionados con hacer efectivo uno de los objetivos primordiales de la educación, la inclusión de todo el alumnado, como término que está relacionado con el acceso, la participación y logros de todos los alumnos, con especial énfasis en aquellos que están en riesgo de ser excluidos o marginados (UNESCO, 2005). Este término implica una reestructuración de la cultura, las políticas y las prácticas de los centros educativos con el objetivo de minimizar las barreras que limitan el aprendizaje y la participación de todo el alumnado (Booth y Ainscow, 2000). Desde que, en 1994, bajo los auspicios de la UNESCO surgiera el nuevo término de "inclusión" en la Declaración de Salamanca (UNESCO 1994), han sido infinitas las definiciones que se han realizado, ya que, en esta declaración, la educación inclusiva es vista como un principio, es decir, como un criterio con peso moral, de carácter orientativo, que no implica compromiso ninguno a sus destinatarios (Echeita y Ainscow, 2011). No es hasta 2005, cuando la UNESCO establece las líneas para la inclusión, apoyadas un año más tarde por la Convención de los Derechos de las Personas con Discapacidad (ONU, 2006), que define un nuevo concepto de inclusión, en el que se dicta que la educación inclusiva es un derecho humano, por lo que todas las personas sin excepción tienen derecho a ella, y por ello, obliga a las autoridades pertinentes a crear las condiciones adecuadas para su disfrute, eliminando las barreras que impidan su ejercicio (Blanco, 2006; Echeita y Ainscow, 2011). Ante esta emergente universalización de los derechos de las personas con discapacidad, España ratifica esta Convención en el BOE número 96, de lunes 21 de abril de 2008 (pág.20648-20659) destacando entre sus 50 artículos aquel expreso dedicado a la previsión de un sistema inclusivo para todas las enseñanzas, el derecho a no ser excluido del sistema educativo general por presentar una discapacidad (artículo 24, pág. 20654) así como el derecho en ese mismo punto (apartado, c) de la realización de "ajustes razonables en función de las necesidades individuales" (pág. 20654). Definiendo a los ajustes razonables como "modificaciones y adaptaciones necesarias y adecuadas que no impongan una carga desproporcionada o indebida, cuando se requieran en un caso particular" (ONU, 2006, pág. 5; citado por Lombana y Báez, 2018), adaptaciones o ajustes como garantía de igualdad y de derecho para el alumnado con discapacidad (Guzmán y Nieto, 2020). Llegados a este punto, existen dimensiones que son de importancia resaltar para comprender el significado funcional de una educación inclusiva (Booth y Ainscow, 2000; Echeita y Ainscow, 2011; Muntaner, 2017) 
y que Echeita y Serrano-Rodríguez (2020) definen recientemente estas 3 como esenciales: Presencia, aprendizaje y participación.

En el que la presencia se refiere al lugar, a donde están escolarizados las y los estudiantes (p.33). Se refiriere a centros educativos regulares, a la necesaria presencia en todo de tipo de actividades de aula, extraordinarias, en el comedor en el patio, destacando la importancia de estar juntos para poder valorar la diversidad humana y entenderla como algo valioso. Por otro lado, la participación, va más allá de la presencia, ya que esta implica aprender con otros y colaborar y cooperar con ellos en el transcurso de las clases y las lecciones (p.34), que precisa de una participación activa y de la preocupación por el bienestar personal y social del alumnado. Esto último, pone en juego el papel que cumplen las emociones y los afectos en la vida escolar del alumnado, junto con importancia de contar con las amistades y grupos de referencia con los que poder compartir y ser parte de actividades significativas de la vida escolar.

El aprendizaje hace referencia a que el alumnado alcance el mejor rendimiento en las diferentes áreas de currículo, relacionando estos dos términos entre sí, para interpretarlos de una manera más comprensiva y que preste más atención al progreso personal sobre el desarrollo de las competencias básicas e imprescindibles y no tanto a la calificación numérica. En este sentido, la educación inclusiva, se entiende como un proceso en el que es necesario articular de forma justa esta tres dimensiones, presencia, participación y aprendizaje. De este modo, resulta necesario abordar las barreras que deben eliminarse para alcanzar este proceso que avance hacia una educación más inclusiva y, en primer lugar, tendremos que identificarlas $y$, en segundo, eliminarlas. Estas barreras las definen Echeita y Serrano-Rodríguez (2020) como el "conjunto de factores (actitudinales, políticas, practicas de aula...) que limitan o puedan limitar la presencia, el aprendizaje y participación, así como el reconocimiento y la valoración de la diversidad del alumnado en un centro escolar determinado" (p.39). Impiden el disfrute del derecho a una educación inclusiva y, para mejorarlo, resulta necesaria la recopilación y evaluación de información para determinar quiénes las experimentan, en qué esferas de su vida académica se sitúan y cuáles son. Por lo que resulta necesario que dupliquemos nuestros esfuerzos, si pretendemos alcanzar una educación de calidad para todos (González-Montesino y Espada, 2020).

La introducción en el aula de las Tecnologías de la Información y la Comunicación (en adelante TIC) en un caso de parálisis cerebral espástica está íntimamente ligado con la eliminación de barreras que suponen el acceso a la comunicación y a la educación en todas sus dimensiones, sociales y emocionales, convirtiéndose en un objetivo explícito, transformando todo el entorno en un recurso. Esto nos acerca, además, al papel tan importante que tienen las familias, realizando inmersiones sociales fundamentales (López, 2018) y persiguiendo la inclusión en el contexto social (Barrón et al., 2019). Además, actualmente, las TIC tienen diferentes funciones cuando estas se aplican a un caso en el que existe una limitación importante en la movilidad, ya que posibilitan el acceso a las áreas del currículo independientemente de las características comunicativo-lingüísticas del alumnado. Se convierten en herramientas personales, fomentando la comunicación con cualquier agente educativo, construyendo un modelo adecuado de identidad y autoestima, creando un espacio de ocio accesible y transformándose en una herramienta social en diferentes contextos $y$, finalmente, se convierten en un instrumento escolar, posibilitando intercambios comunicativos más funcionales (López, 2018; Kochanowicz, 2019).

En el estudio del caso se presenta, la herramienta que permite el acceso a la comunicación y, por tanto, es el paso imprescindible para iniciar cualquier proceso de inclusión en su conjunto, es el lector ocular Iris bond $\mathrm{Duo}^{\circledR}{ }^{\circledR}$, un dispositivo que está íntimamente relacionado con los campos de la psicología cognitiva y la neurociencia (Jiguo et al., 2017). Este hardware se convierte en la llave perfecta para acceder a un entorno inclusivo, proporcionándole al 
alumnado que lo utiliza una forma de comunicarse con el mundo exterior (Salunkhe y Patil, 2016) y, en este caso, el único recurso para comenzar a establecer cualquier tipo de comunicación. El acceso al ordenador a través de la mirada permite el establecimiento de una comunicación fluida y completa tanto a nivel formal como a nivel emocional y puede llegar a ser la única opción efectiva para personas con movilidad muy reducida (García, 2016). El dispositivo lee nuestros movimientos con la unión de cámara infrarroja y otros sensores que posteriormente transforma en señales y coordenadas cartesianas. Se conecta a un ordenador y su software traduce movimientos oculares a señales de introducción de información. Ahora bien, la gestión de estos recursos tecnológicos implica la toma de decisiones, el diseño de acciones con objetivos definidos y docentes dispuestos a implementarlos. Todo este proceso implica activamente a toda la comunidad educativa, responsable en este proceso de transformación cuando un centro educativo se propone integrar las TIC. Por eso, es importante conocer aquellos proyectos de aula como el que aquí se presenta en el que las TIC influyen directa y positivamente para lograr la consecución de los objetivos previstos, así como dar respuesta a los porqués y los para qué de sus aportes. (Zappalá, et al., 2019).

\section{Material y métodos.}

Para realizar la investigación se utilizó el estudio de casos. Se seleccionó ya que el propósito de este va dirigido a la mejora de la realidad educativa a través de situaciones que se producen en la vida real y a partir de un contexto educativo concreto, respondiendo a preguntas del tipo, "cómo" y "por qué" (Yin, 2004). Por otro lado, supone un proceso de investigación-acción colaborativa mediante equipos híbridos de trabajo que busca, principalmente, el desarrollo de prácticas reflexivas con miras a la mejora del aprendizaje y la eficacia docente en un contexto determinado. Mills y Gay (2019) indican que la investigación-acción representa un acercamiento científico a la resolución de problemas educativos de contexto estudiado. Por otro lado, un proceso de investigación colaborativa trasciende a la actual separación entre la teoría y la práctica (Mérida-Serrano et al., 2020) por lo que este tipo de investigaciones educativas con equipos híbridos de trabajo modifica las relaciones tradicionales entre la universidad y la escuela (Sancho-Gil 2007). Como herramientas para la recogida de datos y, a partir de los criterios de evaluación y estándares de aprendizaje, se evaluaron periódicamente exámenes y controles de evaluación y quedaron plasmados al final de cada trimestre de forma cualitativa y a través de rúbricas de evaluación siguiendo la estructura general para todo el grupo de referencia de la alumna, sin distinción alguna (Anexo 1). Estos criterios de evaluación y estándares de aprendizaje se formularon para la consecución de los objetivos de las áreas prescriptivas curriculares además de contemplar aspectos comunicativo-lingüísticos íntimamente relacionados con el uso funcional del equipo que no solo permitía comunicarse a la alumna de forma progresiva y funcional, sino también alcanzar un desarrollo curricular y competencial pleno tal y como marca la legislación actual de nuestro sistema educativo. Igualmente, se recopilaron los datos en situaciones reales mediante la interacción con los agentes educativos que participaron en cada proceso, dotando a todo el proceso educativo de un carácter dinámico y en ocasiones también lúdico, pues el uso de las TIC a través de metodologías de acceso visual confirman su importancia en el recuerdo de los aprendizajes (Cuesta-Cambra et al., 2017), abriendo así el carácter socio-educativo de la investigación, ya que permite analizar y describir comportamientos sociales, percepciones y creencias de las personas (Hernández y Maquilón, 2010). Así, se llevó a cabo un estudio y registro progresivo de los siguientes ítems adoptados por todas las tutorías del centro educativo en el nivel de la alumna en todas las áreas del currículo: "pruebas escritas", "trabajo diario" y "actitud". Estos aspectos tenían asociados los siguientes porcentajes de valoración, consensuados académicamente: $20 \%, 60 \% 20 \%$ de la nota final, respectivamente (Anexo 2). El equipo de trabajo está compuesto por el maestro especialista en 
Audición y Lenguaje del centro, que a su vez es el apoyo permanente dentro del aula de referencia, la tutora del aula, el equipo de orientación del centro, y los directores el Máster en Atención a Necesidades Educativas Especiales en Educación Infantil y Primaria de la Universidad Rey Juan Carlos (miembros del equipo del grupo de investigación "Diversia").

La propia complejidad y extensión del caso, ha supuesto que la denominación de los apartados sufra algún tipo de variación.

\subsection{Caso de estudio}

El objeto de este estudio se centra en RM, una niña con un diagnóstico de Trastorno del Neurodesarrollo (Parálisis Cerebral Infantil con predominio espástico), CIE-11(OMS, 2018)

La parálisis cerebral (PC) se considera como un grupo de trastornos del desarrollo del tono postural y del movimiento, de carácter persistente, que condicionan una limitación de la actividad, que son atribuidos a una agresión no progresiva sobre un cerebro inmaduro (en desarrollo, en la época fetal o primeros años) (Poo, 2008) y que, a su vez, puede acompañarse de otros trastornos (sensitivos, cognitivos, lenguaje, perceptivos, conducta, epilepsia) cuya existencia o no, condicionará de manera importante el pronóstico individual de estos niños. En función de la extensión de la afectación, se denomina bilateral y en el caso de afectar, con igual o mayor afectación, a las 4 extremidades. La Organización Mundial de la Salud (OMS, 2018) la caracteriza, cuando está ligada la espasticidad, por un aumento del tono muscular asociado a reflejos de estiramiento muscular hiperactivos (reflejos tendinosos profundos) y un aumento de la resistencia al estiramiento muscular rápido. Esta situación, se traduce en que RM tiene afectada gravemente su movilidad, no camina de manera autónoma, desplazándose en silla de ruedas $y$, en ocasiones con un andador "Grillo" ${ }^{\circledR}$. No presenta funcionalidad en miembros superiores, y tanto la sedestación (posición básica para permanecer sentado) como el control cefálico son compensados con el uso de diferentes ayudas técnicas. El predominio de la espasticidad supone que no tenga un control en los movimientos voluntarios, como, por ejemplo, el movimiento de los brazos, las manos o masticar. Presenta, además, una disartria severa, que según González y Bevilacqua (2012), es un trastorno marcado por una alteración en la ejecución del movimiento que afecta la producción del habla. Por este motivo, se ve imposibilitada cualquier tipo de expresión oral básica en RM. No obstante, el nivel lingüístico y comprensivo de la niña está en parámetros que en determinadas áreas curriculares supera los marcadores normotípicos, pues a nivel cognitivo tiene todas las capacidades preservadas y es capaz de controlar la mirada de forma funcional. Por lo que nos encontramos con un caso en que la función comunicativa no se puede dar de manera verbal y la comunicación no verbal, especialmente la visogestual, está condicionada por la espasticidad, lo que supone que realizar gestos, por ejemplo, de afirmación o negación también se realizan con gran dificultad y, principalmente, solo pueden ser reconocidos por personas que formen parte de su entorno mas cercano.

En otras palabras, la función comunicativa oral, gestual y escrita no puede establecerse. La niña, que fue matriculada a los tres años en un centro público de escolarización preferente para alumnado con discapacidad motora de la Comunidad de Madrid, comenzó a emplear desde los tres años y de forma gradual diferentes materiales, soportes y Sistemas Alternativos y/o Aumentativos de Comunicación (en adelante SAAC) para acceder a los contenidos curriculares. Familiarizarse con entornos de comunicación digitales, así como con tecnologías de acceso como el lector ocular, provocó que la alumna comprendiera mejor cada herramienta nueva que se introducía en el proceso de enseñanza y aprendizaje, provocando que sus capacidades y potencial cognitivo se mostrara en su totalidad (Hutton, 2019). 
La forma en la que la incidencia de las TIC podía influir positivamente en el proceso de enseñanza y aprendizaje de la alumna, favoreciendo además la inclusión activa en cualquier contexto y ante cualquier agente educativo, hizo que, en los últimos años de la etapa de Educación Infantil, su familia, se implicara al máximo en que su hija pudiera utilizar un sistema de comunicación aumentada que le permitiera este acceso.

Respecto al centro educativo, está situado en Madrid. Es un centro de línea cuatro en todos los niveles de Educación Primaria, salvo en 6o curso, que recoge 5 líneas y de escolarización preferente para alumnado con discapacidad motora (física). Escolariza alumnado desde los 3 hasta los 12 años. En el marco de las concreciones curriculares queda recogida la atención a la diversidad y se refleja en su organización, en sus recursos y en su respuesta adaptada.

\subsection{Estudios de las herramientas de acceso.}

Ante estas circunstancias de motivación e implicación familiar, se estudió el sistema de acceso a la información y la comunicación que fuera más adecuado a sus necesidades y habilidades y que, además, permitiera realizar sus estudios en un centro educativo ordinario, realizando los ajustes necesarios y que no supusieran ningún tipo de adaptación significativa del currículo. Es decir, a partir de la eliminación de las barreras de acceso, tanto físicas (centro ordinario, preferente para alumnado con discapacidad motora), como de acceso a la información y comunicación, la alumna pudiera hacer efectiva la presencia, la participación y el aprendizaje en un entorno que fomentase la igualdad de oportunidades y se concretase en una educación mas inclusiva. En la Tabla 1, se da respuesta al por qué se elige el dispositivo de acceso Irisbond Dúo ${ }^{\circledR}$ $y$, por otro lado, en Anexo 3 se reflejan los motivos de elección de los distintos dispositivos complementarios de software y hardware como recursos TIC fundamentales para desarrollar una actividad docente adecuada: Grid $3^{\circledR}$ y Surface ${ }^{\circledR}$. Destacar la colaboración del Centro de Referencia Estatal de Autonomía Personal y Ayudas Técnicas (Ceapat) como canalizador de información de los diferentes productos disponibles.

\begin{tabular}{lll}
\hline Características fundamentales que debe contener el lector ocular & Irisbond Dúo ${ }^{\circledR}$ & Otros \\
\hline Homologación del Ministerio de Sanidad, Consumo y Bienestar Social & Sí & Sí \\
\hline Ajuste de la distancia de calibración entre la alumna y el ordenador & Sí & No \\
\hline Asistencia técnica ilimitada por parte del fabricante & Sí & No \\
\hline Calibración a través de un solo ojo o de forma binocular & Sí & No \\
\hline Actualizaciones del software del dispositivo sin coste adicional & Sí & No \\
\hline Uso adecuado tanto en exteriores como en interiores & Sí & No \\
\hline Peso, volumen y ajuste adecuado a los dispositivos informáticos & Sí & Sí \\
\hline Buen rendimiento independientemente de las horas del uso & Sí & Sí \\
\hline Calibración entre 0, $1,5,9$ y 16 puntos & Sí & Sí \\
\hline Alimentación USB $2.0,3.0$ & Sí & Ní \\
\hline Tecnología eye tracking por principio de pupila oscura & No & No \\
\hline
\end{tabular}

Tabla 1. Justificación de la elección del lector ocular como medio de acceso al ordenador 
Durante el curso escolar 2018/2019, momento en el que se produjo un salto de etapa educativa de Educación Infantil a la etapa de Educación Primaria y de carácter obligatorio, comenzaron a utilizarse herramientas digitales (tanto "hardware" como "software" específico) de forma sistemática, que no solo beneficiaron el desarrollo cognitivo, social y curricular, tanto en la propia alumna como en su familia, sino también en el resto de alumnado, en el propio centro educativo $y$, en definitiva, en toda la comunidad educativa. Comenzar desde una edad tan temprana con el uso de lectores oculares y SAAC digitales favoreció, sin duda alguna el éxito académico que demostraría después la alumna (McCoy-Thomas, 2019). Además, es a los 6 años y con el paso a etapa obligatoria de Educación Primaria, cuando la alumna puede disponer de la figura de un maestro especialista en Audición y Lenguaje durante toda su jornada escolar, funcionando como puente entre la niña y su sistema, dando alas a un proceso de aprendizaje donde se dan todas las condiciones idóneas para que resulte funcional y pragmático.

\subsection{Objetivos}

Una vez se identifican las necesidades educativas especiales de la alumna, se determinó un sistema de acceso adecuado, ajustado para poder desarrollar al máximo todas sus capacidades cognitivas y comunicativas (Figueredo, 2020) y que pretenden contribuir a alcanzar las máximas competenciales de saber ser, saber hacer y saber estar (Ministerio de Educación y Formación Profesional, 2020), así como a garantizar el máximo desarrollo curricular y, por supuesto, social. No obstante, se plantearon tres tipos de objetivos que se muestran en la Tabla 2, por un lado, los relacionados con el desarrollo curricular y el centro educativo y, por otro, con la aceptación por parte de RM de que las herramientas TICS son su sistema de comunicación en todos los ámbitos de su vida.

\begin{tabular}{|c|c|c|}
\hline Currículo & RM (alumna) & Centro educativo \\
\hline $\begin{array}{l}\text { Desarrollo de competencias en } \\
\text { igualdad de oportunidades, a partir } \\
\text { del currículo común y exclusivamente } \\
\text { con adaptaciones de acceso para la } \\
\text { eliminación de barreras para el acceso } \\
\text { a la comunicación. }\end{array}$ & $\begin{array}{l}\text { Comprender que las Tecnologías de la } \\
\text { Información y la Comunicación son el } \\
\text { medio que le permiten acceder a los } \\
\text { contenidos del currículo de Educación } \\
\text { Primaria }\end{array}$ & $\begin{array}{l}\text { Emplear de forma satisfactoria una } \\
\text { metodología de acceso visual para } \\
\text { acceder a las Tecnologías de la } \\
\text { Información y la Comunicación }\end{array}$ \\
\hline $\begin{array}{l}\text { Alcanzar una comunicación funcional } \\
\text { (Habilidades Metalingüisticas: } \\
\text { omisiones, sustituciones) }\end{array}$ & $\begin{array}{l}\text { Usar un soporte informático en } \\
\text { cualquier contexto para que le permita } \\
\text { generalizar los aprendizajes que } \\
\text { adquiera dentro del aula de referencia }\end{array}$ & Cooperación Docente \\
\hline $\begin{array}{l}\text { Mejorar las habilidades } \\
\text { metalingüísticas para que no se } \\
\text { produzcan omisiones y sustituciones } \\
\text { de letras o sílabas en palabras de } \\
\text { estructura compleja }\end{array}$ & $\begin{array}{l}\text { Mejorar el uso funcional del equipo } \\
\text { (diseño basado en la evidencia) }\end{array}$ & $\begin{array}{l}\text { Entorno de aprendizaje y Cultura } \\
\text { inclusiva }\end{array}$ \\
\hline $\begin{array}{l}\text { Desarrollar una buena comprensión } \\
\text { lectora, siendo capaz de contestar a } \\
\text { preguntas de corte literal e inferencia }\end{array}$ & & $\begin{array}{l}\text { Interés por las TIC's como recurso } \\
\text { para fomentar la calidad y la } \\
\text { inclusión de todo el alumnado. }\end{array}$ \\
\hline \multicolumn{3}{|l|}{ Comunicación Social } \\
\hline Presencia, participación y aprendizaje & Presencia, participación y aprendizaje & $\begin{array}{l}\text { Presencia, participación y } \\
\text { aprendizaje }\end{array}$ \\
\hline
\end{tabular}

Tabla 2 Objetivos currículo - alumna - centro educativo 


\subsection{Adaptaciones de acceso al currículo y proceso de inclusión}

Una vez recogida toda la información necesaria, además de mantener entrevistas con la familia así como diversas reuniones con el equipo de atención a la diversidad del centro educativo, los equipos de coordinación de etapa y de nivel educativo además de con el servicio de orientación educativa y Universidad, no se realizaron adaptaciones curriculares significativas en la mayoría de las áreas contempladas en el Currículo Oficial (solamente en el área de Educación Física) debido a la capacidad cognitiva de la alumna y a su desempeño, plasmado de forma objetiva en la documentación anexa a su expediente. Por lo tanto, se continuó con adaptaciones de acceso al currículo a través de soportes tecnológicos y, dependiendo de la estructura interna de cada área curricular, se elaboró una respuesta educativa diferente. En algunas materias fue suficiente con introducir los contenidos en su comunicador, otras como Matemáticas supusieron la elaboración de material propio (López, 2018).

Las adaptaciones de acceso al currículo se realizaron a partir de las clasificaciones de Arnaiz (2000), y resultan imprescindibles para generar un proceso de enseñanza y aprendizaje adecuado, dirigido al alumnado que presenta necesidades educativas especiales y a través de un proceso de modificación realizado sobre el currículo ordinario, siendo estas: personales, espaciales, materiales y de comunicación (Anexo 4). Así, se aplicó para la alumna tanto la programación general de aula como la programación específica del maestro especialista en Audición y Lenguaje, donde quedaron recogidos todos los objetivos, contenidos, recursos (materiales, espaciales y por supuesto, personales), metodología de enseñanza, criterios de evaluación y estándares de aprendizaje. Algunas de las herramientas digitales empleadas en el proceso de enseñanza y aprendizaje y que resultaron imprescindibles fueron las siguientes: EyeHarp, Drawboard PDF, Adobe Acrobat Reader DC, Creating music - Block games y, por supuesto, el "software" de comunicación que le permitió un acceso sin límites a todo su mundo: Grid3, software específico de comunicación aumentativa que permite un manejo fluido y un control de edición ilimitado por parte del docente (García y Marín, 2016). Respecto al uso de este sistema requiere de un aprendizaje y, por tanto, de un nivel de especialización importante por parte también docente. Para su puesta en marcha se requiere de el empleo de un brazo mecánico articulado, un lector ocular, un ordenador portátil convertible y un "software" específico de comunicación aumentativa. Una vez encendido su ordenador hay que calibrar el ratón visual con la mirada de la niña, conocer las herramientas que ofrece, ajustar los tiempos, la cantidad de estímulos que aparecen en pantalla, enseñar a la alumna dónde tiene que mirar para simular el doble clic de un ratón convencional, movimientos de arrastre y selección y un sinfín de configuraciones que para otras personas pasan desapercibidas por poder usar periféricos sin dificultad alguna. Después de la calibración, se accede a un programa informático para la comunicación y el acceso que resulta extremadamente útil para la alumna a la hora de emplear texto. Le permite la comunicación cara a cara, la comunicación remota mediante correo electrónico para enviar los deberes realizados semanalmente, así como el acceso al ordenador de forma adaptada a sus necesidades.

\section{Análisis y resultados.}

La alumna, desde el primer momento en el que se implementó el uso de un sistema de comunicación y de acceso al currículo en el aula, mostró resultados favorables, aunque no fue así durante todo el curso escolar. Al principio del mismo quería utilizar las mismas herramientas de trabajo que sus compañeros, siendo necesario utilizar la negociación para que, poco a poco, fuese siendo consciente de la importancia del uso de las TIC y cómo estas repercutirían en su desarrollo curricular y social, llegando, finalmente a utilizar estas nuevas tecnologías no solo en el aula, sino en el resto de contextos del centro educativo, como en el espacio destinado a actividades físicas y deportivas o de descanso y recreo, creando un programa de patios adaptado 
donde todo el alumnado podía participar de la misma forma. Esto es importante remarcarlo, pues ha contribuido muy positivamente a la consecución de los objetivos planteados en el estudio.

Ya desde la primera evaluación y hasta el final del curso escolar, la alumna consiguió alcanzar todos los objetivos planteados en el área de Audición y Lenguaje, dejando en progreso los dos últimos, es decir, "Mejorar las habilidades metalingüísticas para que no se produzcan omisiones y sustituciones de letras o sílabas en palabras de estructura compleja" y "Desarrollar una buena comprensión lectora, siendo capaz de contestar a preguntas de corte literal e inferencial". Los aspectos relacionados con la adquisición de la lectura y de la escritura y, en especial, leer y escribir sílabas de estructura compleja o adecuar la atención a la hora de leer todas las palabras (con sentido y sin él) que conforman una oración completa, es algo que le resultaba complejo. Para solventar este tipo de hándicap se utilizó "software" específico que ayudó a mejorar estos aspectos, tales como Lectura de Textos 3 o Katalekto, que ofrecen "feedback" auditivo y apoyo visual en determinadas unidades gráficas, respectivamente. Este tipo de "software" se empleó en el aula para todo el alumnado, no solo para la alumna, repercutiendo positivamente en su competencia lecto-escritora.

En cuanto a los objetivos planteados en la programación general de aula, desde el primer momento la alumna los alcanzó, situándose en puntuaciones por encima de la media de su grupo de referencia. Las calificaciones en las áreas de "Matemáticas", "Ciencias Sociales", "Ciencias de la Naturaleza" y "Educación Plástica" arrojaron puntuaciones de 10 sobre 10. En la asignatura de "Lengua Castellana y Literatura" y debido a sus dificultades en conciencia fonológica y comprensión lectora inferencial, ésta se situó en 9,60 puntos, nuevamente sobre 10. En el segundo trimestre, las calificaciones en las áreas de "Matemáticas" y "Lengua Castellana y Literatura" descendieron ligeramente en 3 y 1 punto respectivamente, en el apartado de "prueba escrita". Esto es causado por dos aspectos: en primer lugar por la complejidad de los enunciados a los que la alumna se enfrenta y cuyo proceso se encuentra envuelto por los procesos de acceso y procesamiento relacionados con la lectura y la escritura $y$, en segundo lugar, por la mejoría del manejo de su sistema de comunicación, aumentando parejamente con el desempeño social, provocando que interaccione más con sus compañeros y compañeras durante las clases, provocando que haya periodos de atención de menor calidad. Finalmente, en el tercer trimestre, sus calificaciones en todas las áreas mejoraron hasta alcanzar puntuaciones que se situaron en rangos de entre 9 y 10 puntos. La alumna manejaba ya con mayor soltura su sistema, superando paulatinamente sus dificultades relacionadas con la lectura y la escritura e integrando que la comunicación, como el resto de sus compañeros y compañeras, tiene sus momentos, respetando los turnos comunicativos y adaptando las funciones del lenguaje a los diferentes contextos.

Tanto en el segundo como en el tercer trimestre, los logros académicos de la alumna también mejoraron en los aspectos anteriormente referenciados al mismo tiempo que lo hacía en el manejo de las TIC, de su sistema de comunicación y del lector ocular que le permitía el acceso a todas las herramientas digitales con las que seguir el ritmo académico y social del centro educativo. Lo mismo sucedió con sus compañeros y compañeras, pues convivían con el sistema de la alumna de una forma activa, aprendieron interaccionar con él y con ella, integrándolo en cualquier actividad de una forma natural, al igual que el resto de TIC que se utilizaban en el aula.

\section{Discusión y conclusiones.}

Para poder alcanzar los objetivos del currículo de Educación Primaria la alumna ha empleado recursos materiales especiales y son estos los que requieren una especialización remarcable. El uso del lector ocular en el proceso de enseñanza y aprendizaje ha conseguido que, tanto el 
maestro especialista en Audición y Lenguaje como la tutora del aula mejoren la calidad de su práctica docente al mejorar el marco de la misma, demostrando que el uso de tecnologías de seguimiento ocular en el campo de la educación mejora los estándares educativos (Sun, et al., 2018). Utilizar el lector ocular con la alumna de manera sistemática, no solo mejoró su nivel de desarrollo y competencia curricular, sino aspectos cognitivos fundamentales para poder utilizar este sistema tales como la atención y la memoria visual en tareas de selección de estímulos visuales, incluso por encima del resto de sus compañeros, demostrando que este tipo de tecnologías son un poderoso soporte para desarrollar habilidades cognitivas en el alumnado (Amantis et al., 2011). Además, actualmente la alumna se comunica con todo este conjunto de herramientas, favoreciendo su desarrollo social y emocional y no solo académico. El maestro especialista que la ha apoyado ha conseguido tejer una auténtica red socioemocional entre ella y sus compañeras y compañeros, cohesionando y estrechando las relaciones entre los discentes. No obstante, la niña sigue necesitando este apoyo para que poco a poco vaya generalizando los aprendizajes y sea cada vez más autónoma, actualmente aún es pronto para retirar un apoyo de tales características. La alumna es excepcional y como tal, necesita un recurso excepcional (Marwah y Kharb, 2019). No debemos pasar por alto la gran influencia que ha tenido en el desarrollo de la alumna la colaboración e implicación tan estrecha, cercana y continuada que ha mantenido el centro educativo con la familia de la niña. Para conseguir el éxito de este proceso de inclusión, es necesaria una mediación familiar óptima, acabando con las barreras de la brecha digital, procurando que unas competencias digitales mínimas se instauren en la familia del alumnado (López-Sánchez y García del Castillo, 2017). Así, desde el primer momento de la escolarización de la alumna, la coordinación ha sido fundamental para que se utilizara de una forma bidireccional este tipo de tecnología, favoreciendo su uso en cualquier contexto y permitiendo a la alumna (y también al alumnado) a integrarlo como algo realmente natural y necesario en un proceso absolutamente inclusivo. Podemos afirmar que el uso continuado de las TIC y, en este caso, de los lectores oculares como acceso a un SAAC y a distintos "softwares" que permiten la lectura, la expresión escrita, y/o la interacción musical y plástica, ha contribuido a que el centro educativo, siempre con una actitud abierta hacia el uso de las TIC (Macià, 2016), ha ofrecido recursos ajustados a las necesidades del alumnado, conectando con todos los agentes educativos, generando contextos óptimos donde la inclusión y el aprendizaje van de la mano.

Está claro que las dificultades que surgen de la limitación en la movilidad afectan a las oportunidades de aprendizaje de la alumna, pero gracias al uso de un comunicador digital y el sistema de acceso basado en un lector ocular, estas se ven minimizadas hasta el punto en el que estas diferencias desaparecen, produciéndose un aprendizaje más eficiente (Molina et al., 2017). El desarrollo y la puesta en práctica de la competencia digital ha permitido que el alumnado adquiera actitudes y valores positivos, adaptándose a las nuevas necesidades establecidas por las tecnologías, apropiándose de ellas y adaptándolas a sus propios fines y a la capacidad de interaccionar socialmente en torno a ellas. El abordaje de este estudio desde la competencia digital ha desarrollado en los discentes una actitud activa hacia las tecnologías y sus medios, valorando y respetando su uso, provocando la participación y el trabajo cooperativo, así como aumentando la motivación y la curiosidad por el aprendizaje y la mejora en el uso de las TIC (Cummins, 2017).

Gracias al uso de las TIC de acceso visual disponibles en el aula y en el centro educativo (sumando la Pizarra Digital Interactiva al propio sistema de "eye tracking") tanto la alumna objeto de estudio como sus compañeros y compañeras fueron adquiriendo y manifestando gradualmente un crecimiento que les proporcionó las herramientas necesarias para asimilar y poder participar en el contexto educativo inclusivo, además de adquirir la capacidad para enfrentarse a nuevos modelos de pensamiento que les serán de utilidad en el desarrollo de nuevas competencias a 
nivel social y académico, las cuales le serán de suma importancia a lo largo de su vida (Soprano, 2001).

A partir del estudio de este caso, se ha podido valorar en profundidad como el uso de tecnologías de barrido ocular, junto con el uso de recursos TIC como sistema de acceso a la comunicación, y al currículo, ha tenido repercusiones sobre la vida diaria de una alumna con parálisis cerebral espástica en todos los ámbitos, destacando el proceso de inclusión educativo, familiar, personal, cognitivo, lingüístico, emocional y social que, en este contexto, se ha traducido en un proceso de educación inclusiva real, con presencia, participación y aprendizaje. Por lo que dicho trabajo puede servir de referente a otros centros que se encuentren en la misma situación, así como servir de formación a los docentes y orientar la reflexión hacia los cambios educativos necesarios que permitan que las escuelas representen un marco favorable para asegurar la igualdad de oportunidades, favoreciendo así una sociedad más inclusiva, justa y democrática.

\section{Referencias Bibliográficas}

Amantis, R., Corradi, F., Molteni, A., Massara, B., Orlandi, M., Federici, S., Belardinelli, M. y Mele, M.L. (2011). Eye-tracking assistive technology: Is this effective for the developmental age? Evaluation of eye-tracking systems for children and adolescents with cerebral palsy. Assistive Technology Research Series. 29. 489-496. https://doi.org/10.3233/978-1-60750-814-4-489

APA, (2013). DSM-5, Manual diagnóstico y estadístico de los trastornos mentales. Masson.

Blanco, G. (2006). La equidad y la inclusión social: uno de los desafíos de la educación y la escuela hoy. Reice: Revista Electrónica Iberoamericana sobre Calidad, Eficacia y Cambio en Educación, 4(3), 115. http://links.uv.es/xGM2AjL

Barrón, E.O., de Luna, R. y González, M.Z. (2019). Eye tracking technology as an Alternative of Communication for disabled people. Revista Iberoamericana de producción académica y gestión educativa. 6 (12), 1-14. http://pag.org.mx/index.php/PAG/article/view/819

BOE, número 96 de 21 de abril de 2008. 6963. Jefatura del Estado. https://www.boe.es/boe/dias/2008/04/21/pdfs/A20648-20659.pdf

Booth, T. y Ainscow, M. (2000): Índice de Inclusión. Centro de estudios en educación. Bristol.

Cummins, R.G. (2017). Eye Tracking. En Matthes. C.S. Davis and R.F. Potter (Ed.). The International Encyclopedia of Communication Research Methods (1-9). Texas Tech University, USA. https://doi.org/10.1002/9781118901731.iecrm0099

Cuesta-Cambra, U., Niño-González, J.I. y Rodríguez-Terceño, J. (2017). El procesamiento cognitivo en una app educativa con electroencefalograma y "Eye Tracking". Comunicar, 52(25), 41-50. https://doi.org/10.3916/C52-2017-04

Echeita-Sarrionandía, G., y Ainscow, M. (2011). La educación inclusiva como derecho: marco de referencia y pautas de acción para el desarrollo de una revolución pendiente, Tejuelo: Revista de Didáctica de la Lengua y la Literatura 12, 26-46. https://repositorio.uam.es/handle/10486/661330

García, I. (2016). ELA Andalucía: Irisbond, nuevo sistema para la comunicación alternativa. Consultado el 10 de marzo de 2020. http://www.elaandalucia.es/WP/irisbond-nuevo-sistema-para-lacomunicacion-alternativa/

García, L. y Marín, D. (2016). TIC y Educación Especial: intervención con The Grid 2 en un caso de Parálisis Cerebral. Revista DIM: Didáctica, Innovación y Multimedia, (34). Octubre. https://ddd.uab.cat/record/166504

González-Montesino, R. y Espada-Chavarría, R. (2020). Espacios universitarios de aprendizaje inclusivos, bilingües y multimodales: el caso del alumnado sordo signante. Revista De Estilos De Aprendizaje, 13(25), 70-83. http://revistaestilosdeaprendizaje.com/article/view/1519 
González R. A. y Bevilacqua, J. A. (2012). Las Disartrias. Revista Hospital Clínico Universidad de Chile. 2012. (23), $299-309$

Guzmán, R. y Nieto, A.B. (coord..). (2020) Políticas públicas en defensa de la inclusión, la diversidad y el género. Salamanca: Nueva Graficesa, S.L.

Hernández-Pina, F. y Maquilón-Sánchez, J.J. (2010). Las concepciones de la enseñanza. Aportaciones para la formación del profesorado. Revista Electrónica Interuniversitaria de Formación del Profesorado, $13(3), 17-25$.

Hutton, S. (2019). Eye Tracking Methodology. En C. Klein y U. Ettinger (Ed.), Eye Movement Research (pp. 277-308). Springer Nature Switzerland. https://doi.org/10.1007/978-3-030-20085-5 8

Irisbond (2017). Irisbond Dúo. [dispositivo]. https://www.irisbond.com/

Jiguo X., Cheng Q., Chunyong L., Jingwei Y. y Chenggang Z. (2017). A crucial temporal accuracy test of combining EEG and Tobii eye tracker. Medicine, 96 (13), 1-11. https://doi.org/10.1097/MD.0000000000006444

Kochanowicz, A.M. (2019). Eye-Tracking in the Diagnosis, Therapy and Education of Children with Multiple Disability: An Outline of Issues. Elementary education in theory and practice. 4(54), 109-119. https://www.ceeol.com/search/article-detail?id=848847

Lombana, O.I. y Báez. C.I. (comp.). (2018) Didácticas para la vida: Reflexiones desde una educación geográfica multidimensional e inclusiva. Universidad de Pedagogía Nacional de Bogotá.

López-Sánchez, C. y García del Castillo, J. A. (2017). La familia como mediadora ante la brecha digital: repercusión en la autoridad. Revista Colombiana de Ciencias Sociales, 8(1), 108-124. http://dx.doi.org/10.21501/22161201.1928

López, M.L. (2018). Respuesta TIC para un alumno con parálisis cerebral, escolarizado en centros ordinarios: Viaje a través de toda la educación obligatoria. En Escola Superior de Educação de Paula Frassinetti (Ed.), (pp. 641-649). A Fenda Dixital: TIC, NEAE, Inclusión e Equidade. Fenda Digital. http://repositorio.esepf.pt/handle/20.500.11796/2637

Macià, M. (2016). La comunicación familia-escuela: el uso de las TIC en los centros de primaria. Revista Electrónica Interuniversitaria de Formación del Profesorado, 19(1), 73-83. http://dx.doi.org/10.6018/reifop.19.1.245841

Marwah, N. y Kharb, L. (2019). Eye tracking: A new evolution in technology. International Journal of Engineering Applied Sciences and Technology. 04 (7), 252-254. https://doi.org/10.33564/IJEAST.2019.v04i07.043.

McCoy-Thomas, T. (2019). Eye Tracking and Learning Predictability. Journal of International Education and Practice. 2(4). 18-28. https://doi.org/10.30564/jiep.v2i4.1632

Mérida-Serrano, R., González-Alfaya, M. E., Olivares-García, M. A., Rodríguez-Carrillo, J., y Muñoz-Moya. (2020). El Prácticum, un Espacio para la Investigación Transformadora en los Contextos Educativos Infantiles. Revista Iberoamericana sobre Calidad, Eficacia y Cambio en Educación. 18(2), 17-34. https://doi.org/10.15366/reice2020.18.2.001

Microsoft Corporation (2015). Surface 4 Pro. [dispositivo]. https://support.microsoft.com/eses/help/4023446/surface-pro-4-features

Mills, G. E., y Gay, L. R. (2019). Educational research: competencies for analysis and applications. London: Pearson.

Ministerio de Educación y Formación Profesional (2020). LOMCE: Competencias clave. Madrid: Gobierno de España. http://www.educacionyfp.gob.es/educacion/mc/lomce/curriculo/competenciasclave/competencias-clave.html

Molina, A., Navarro, O., Lacruz, M. y Ortega, M. (2017). El empleo de técnicas de seguimiento ocular para evaluar materiales educativos en Educación Primaria. Revista de Educación, 376. 87-109. https://doi.org./10.4438/1988-592X-RE-2017-376-345 
Organización Mundial de la Salud (OMS), 2018. CIE-11. Clasificación Internacional de Enfermedades, 11a revisión. https://icd.who.int/es

Poo, P. (2008). Parálisis cerebral infantil. Asociación Española de Pediatría. Servicio de Neurología. Hospital Sant Joan de Dèu, Barcelona. https://www.aeped.es/sites/default/files/documentos/36pci.pdf

Ramos-Elizondo, A., Herrera-Bernal, J., y Ramírez-Montoya, M. (2010). Developing cognitive skills with mobile learning: A case study. [Desarrollo de habilidades cognitivas con aprendizaje móvil: Un estudio de casos]. Comunicar, 34, 201-209. https://doi.org/10.3916/C34-2010-03-20

Salunkhe P. y Patil, A.R. (2016). A device controlled using eye movement. International Conference on Electrical, Electronics, and Optimization Techniques (ICEEOT), Chennai, 732-735, https://doi.org/10.1109/ICEEOT.2016.7754779

Sancho-Gil, J. M. (2017). Discursos y prácticas en torno a las competencias en educación. Fonseca, Journal of Communication, 15, 127-144. https://doi.org/10.14201/fjc201715127144

SmartBox (2018). Grid3. (versión 3.0.30). [software] https://thinksmartbox.com/product/grid-3/

Soprano, A. M. (2001). La "hora del juego". Lingüística. Disfasias, afasias, autismo, evaluación, orientación. Argentina: Lumiere.

Sun Y., Li Q., Zhang H., Zou J. (2018) The Application of Eye Tracking in Education. In: Pan JS., Tsai PW., Watada J., Jain L. (eds) Advances in Intelligent Information Hiding and Multimedia Signal Processing. IIH-MSP 2017. Smart Innovation, Systems and Technologies, vol 82. Springer, Cham. https://doi.org/10.1007/978-3-319-63859-1_4

UNESCO (2005): Guidelines for inclusión: Ensuring Access to Education for All. UNESCO.

Yin, R. (2004). How to do better case studies with illustrations from 20 exemplary case studies. En Leonard B. y Debra J.R. (Ed.). Applied social research methods (2a. ed.) (pp. 254-283). Sage Publications, Inc: United States of América. https://dx.doi.org/10.4135/9781483348858

Zappalá, D., Köppel, A. y Suchodolski (2019). Inclusión de tic en escuelas para alumnos con discapacidad intelectual. Ministerio de Educación de la Nación. 
Anexo 1. Ejemplo de rúbrica de evaluación de exámenes y controles - Lengua Castellana y Literatura. España, M.; Gallego, V.; Luna, S.; Parejo, A. y Ríos, S. (2017). Lengua +. 1o de Primaria. Madrid: Santillana.

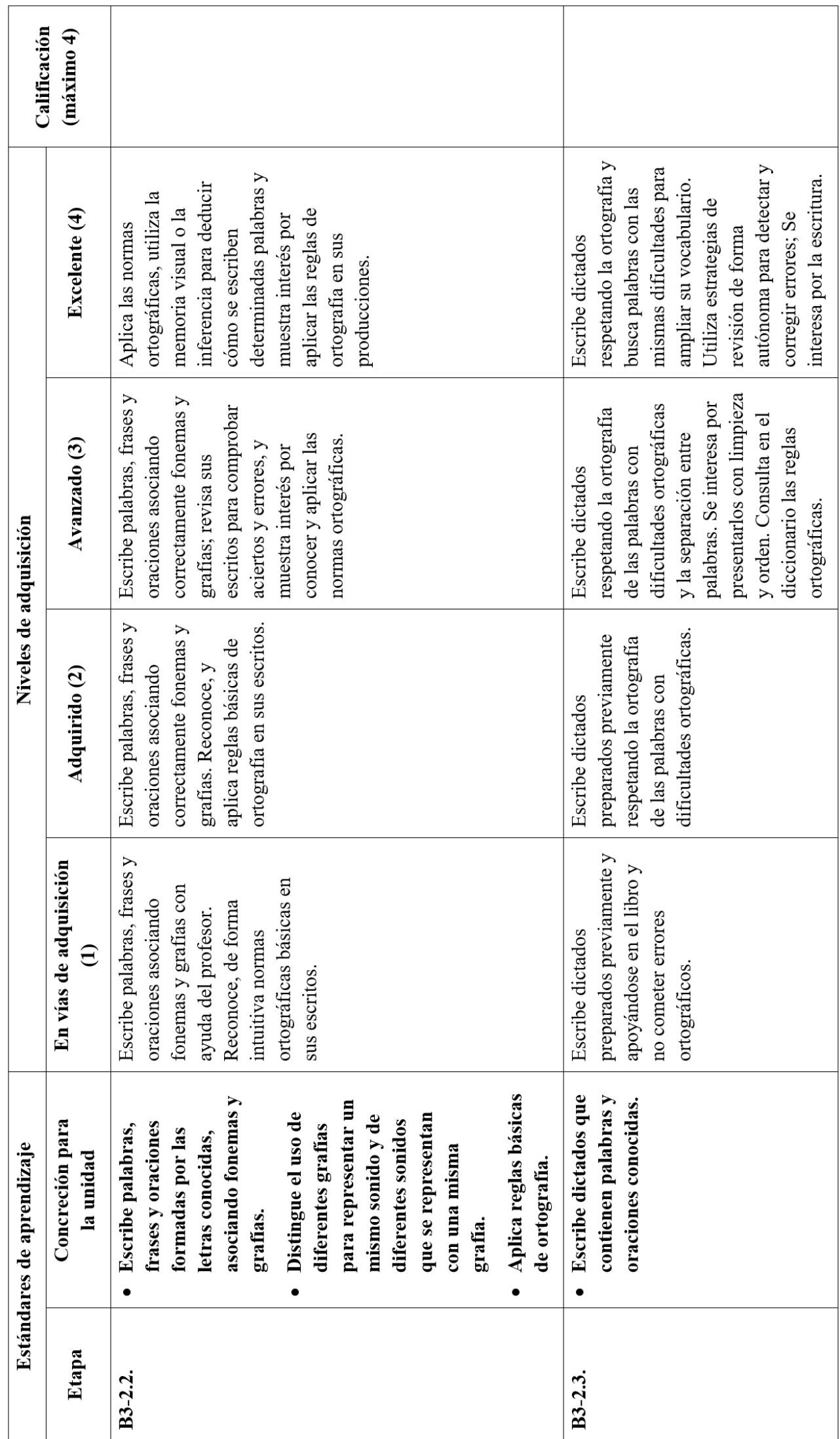




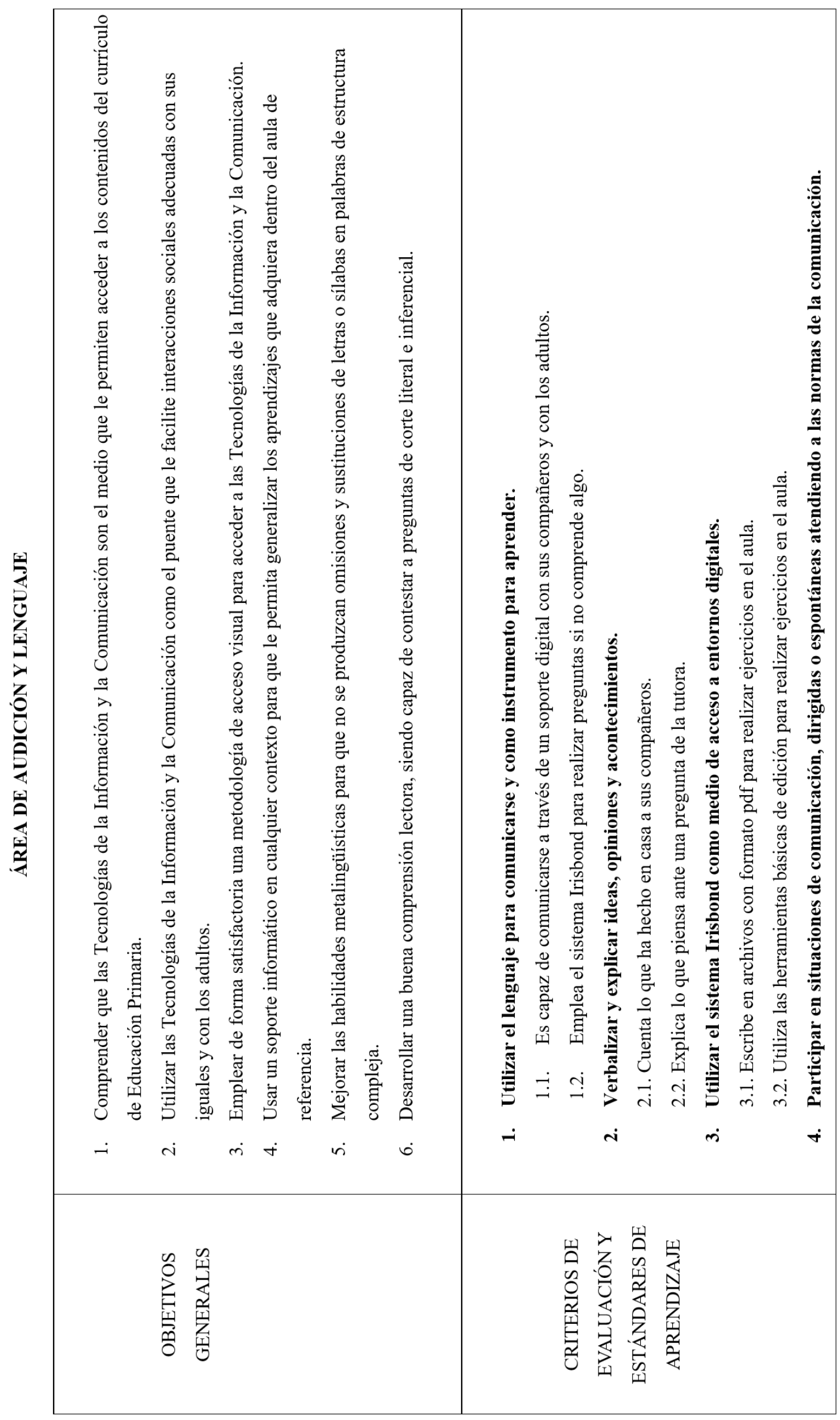




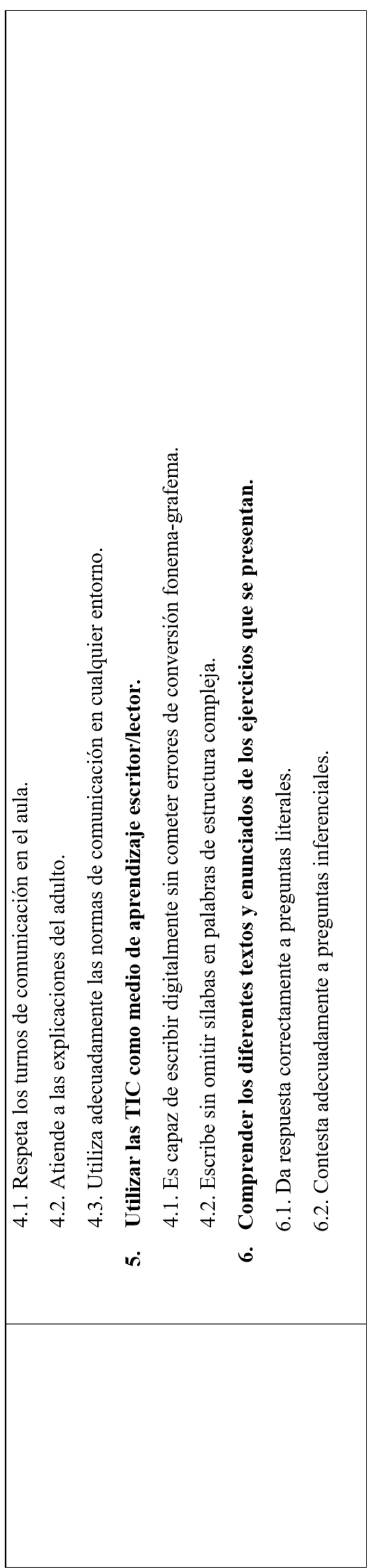


Anexo 2. Rúbrica de calificaciones trimestrales.
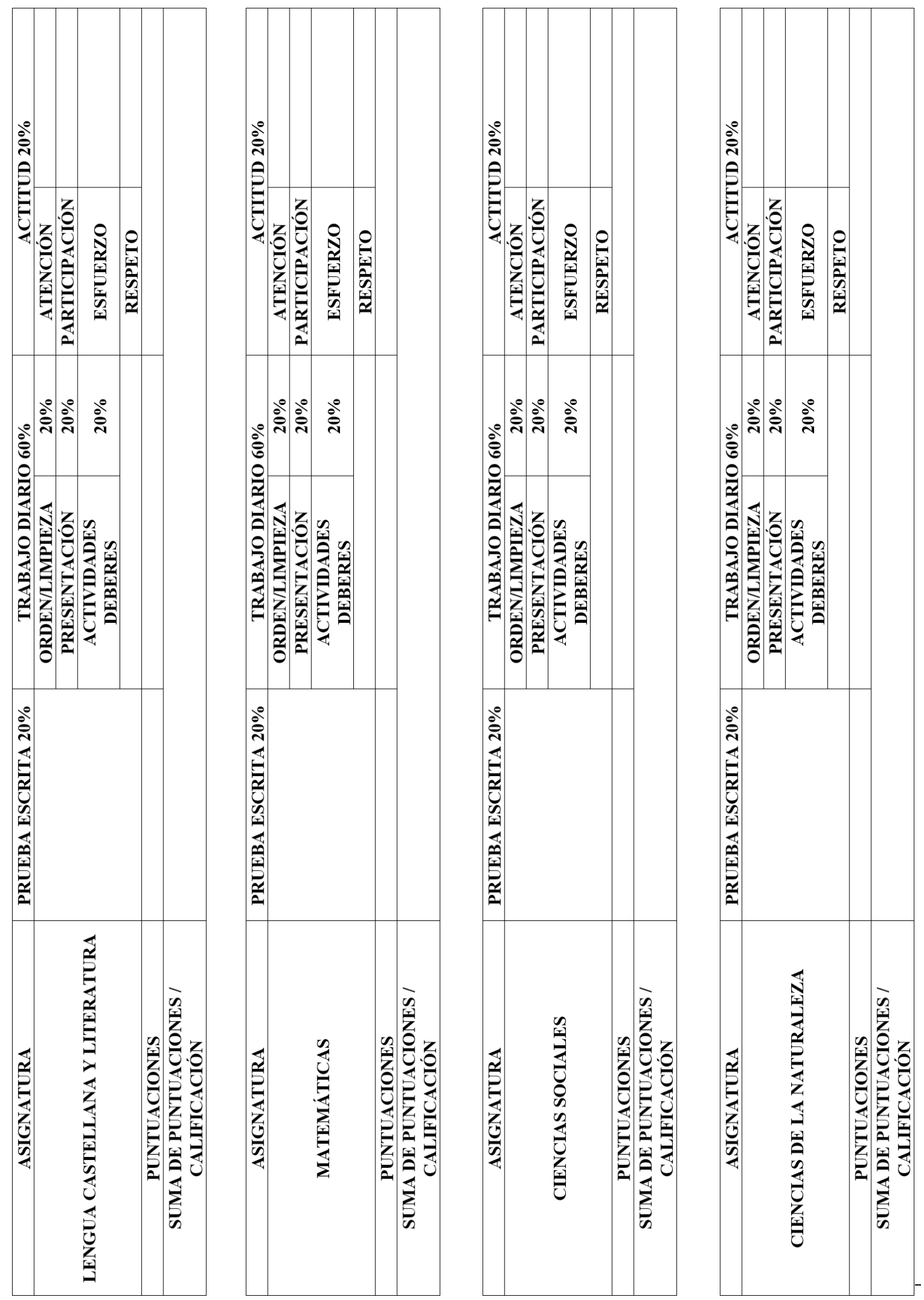
Anexo 3. Justificación de la elección del software de comunicación alternativa.

\begin{tabular}{|l|c|c|}
\hline \multicolumn{3}{|c|}{ Justificación de la elección del Software de Comunicación Alternativa } \\
\hline Características fundamentales que debe contener el software & Grid3 ${ }^{\circledR}$ & Otros \\
\hline Combinación de texto y pictogramas & Sí & Sí \\
\hline Librerías pictográficas propias con más de 20000 símbolos & Sí & No \\
\hline Predicción de texto con tecnología “Switkey" & Sí & No \\
\hline Conjugación verbal para pictogramas & Sí & No \\
\hline Historial de frases utilizadas & Sí & No \\
\hline Acceso a Windows optimizado para usuarios de mirada & Sí & No \\
\hline Gestor de documentos integrado & Sí & No \\
\hline Acceso a nuevos paquetes de cuadrículas en línea & Sí \\
\hline $\begin{array}{l}\text { Posibilidad de emparejarlo con un teléfono y controlarlo desde la } \\
\text { aplicación }\end{array}$ & Sí & No \\
\hline $\begin{array}{l}\text { Posibilidad de escuchar los correos electrónicos y SMS que se } \\
\text { reciben }\end{array}$ & Sí & No \\
\hline Posibilidad de edición remota de los paquetes de cuadrículas. & Sí & No \\
\hline $\begin{array}{l}\text { Paquetes para la comunicación diseñados para personas con } \\
\text { capacidad de lectoescritura }\end{array}$ & Sí & No \\
\hline $\begin{array}{l}\text { Paquetes con veinticinco actividades interactivas con } \\
\text { vocabularios asociados para divertirse e iniciarse en la } \\
\text { comunicación }\end{array}$ & Sí & No \\
\hline $\begin{array}{l}\text { Paquetes de cuadrículas para acceder a otras aplicaciones del } \\
\text { ordenador con la mirada }\end{array}$ & Sí & So \\
\hline Paquetes de cuadrículas para controlar el entorno & No \\
\hline Capacidad de almacenar mensajes personalizados & No \\
\hline $\begin{array}{l}\text { Inclusión de una propia herramienta digital de calibración de la } \\
\text { mirada }\end{array}$ & Sí \\
\hline Compatible con SO Windows e iOS & \\
\hline
\end{tabular}

\begin{tabular}{|l|c|c|}
\hline \multicolumn{2}{|c|}{ Justificación de la elección del ordenador que contenga el software } \\
\hline $\begin{array}{l}\text { Características fundamentales que debe presentar el equipo } \\
\text { informático }\end{array}$ & Surface ${ }^{\circledR}$ & Otros \\
\hline Pantalla de más de 10 pulgadas & Sí & Sí \\
\hline Peso soportable por un brazo articulado (autonomía) & Sí & No \\
\hline Procesador 1.33GHz o más rápido & Sí & Sí \\
\hline Memoria de, como mínimo, 2GB RAM & Sí & Sí \\
\hline Disco duro con más de 3GB libres & Sí & Sí \\
\hline Sistema operativo Windows 7, 8 o 10 & Sí & Sí \\
\hline Conexión Wi-Fi y Bluetooth & Sí & Sí \\
\hline Adaptable a suporte para tableta u ordenador convertible & Sí & Sí \\
\hline Sensor de luz ambiental & Sí & No \\
\hline
\end{tabular}


Anexo 4. Adaptaciones en los elementos de acceso al currículo. Adaptación de Arnaiz (2000)

\begin{tabular}{|c|c|}
\hline \multicolumn{2}{|c|}{ Adaptaciones en los elementos del acceso al currículo. Adaptación de Arnaiz (2000). } \\
\hline Personales & $\begin{array}{l}\text { - Se facilita la incorporación de apoyos a través de las siguientes figuras: } \\
\text { Maestro Especialista en Audición y Lenguaje con atención directa y exclusiva } \\
\text { hacia la alumna; Fisioterapeuta que, además de realizar las sesiones } \\
\text { prescriptivas, apoya a la alumna en las sesiones del área de Educación Física; } \\
\text { Técnico en Integración Social, interviniendo a través del Programa de Patios } \\
\text { Escolares y Técnicos de Atención Educativa III que lleva a la práctica } \\
\text { adaptaciones posturales fundamentales para favorecer el acceso al } \\
\text { currículo. } \\
\text { - Para modificar las actitudes de la comunidad docente respecto a las } \\
\text { necesidades educativas especiales se llevan a cabo actividades formativas } \\
\text { sobre el manejo y funcionamiento del sistema de comunicación de la } \\
\text { alumna, así como jornadas en la etapa de Educación Primaria dentro del } \\
\text { marco del área de Educación Física sobre diferentes deportes adaptados. } \\
\text { - Estableciendo reuniones periódicas del equipo de Atención a la Diversidad } \\
\text { con el claustro y las familias implicadas en el proceso de enseñanza y } \\
\text { aprendizaje se han ajustado las expectativas respecto al alumnado con } \\
\text { necesidades educativas especiales. }\end{array}$ \\
\hline Espaciales & $\begin{array}{l}\text { - Al tratarse de un centro público de escolarización preferente para } \\
\text { alumnado con discapacidad motora, todas las barreras arquitectónicas } \\
\text { quedan suprimida en cualquiera de sus formas. } \\
\text { - Gracias al uso de apoyos visuales pictográficos, imágenes reales y texto } \\
\text { escrito en la lengua vehicular, existen indicadores que facilitan la } \\
\text { localización de los diferentes espacios, así como la orientación y localización } \\
\text { de los mismos. } \\
\text { - Para compensar las limitaciones de la alumna y favorecer su participación, } \\
\text { se ubicó a la alumna cerca de la tutora del aula de referencia, en contra de } \\
\text { luz solar y al mismo tiempo, cerca de una fuente de alimentación } \\
\text { permanente para su sistema informático, favoreciendo al mismo tiempo su } \\
\text { movilidad y autonomía. }\end{array}$ \\
\hline Materiales & $\begin{array}{l}\text { - El departamento de Fisioterapia junto al centro educativo facilita de forma } \\
\text { permanente mobiliario adaptado: bipedestador, mesa con escotadura, atril, } \\
\text { agarradera para el miembro superior derecho, lámina antideslizante para el } \\
\text { material de mesa y material escolar adaptado mediante engrosadores y } \\
\text { material fabricado a tal efecto. } \\
\text { - El centro educativo proporcionó un ordenador portátil desde la etapa de } \\
\text { Educación Infantil con un software de comunicación basado en el uso de } \\
\text { pictogramas y texto, adaptando, además, los contenidos curriculares desde } \\
\text { el primer momento en que la alumna se escolarizó. }\end{array}$ \\
\hline
\end{tabular}


Adaptaciones en los elementos del acceso al currículo. Adaptación de Arnaiz (2000).

- Desde la etapa de Educación Infantil, la alumna asistió a sesiones, tanto de Audición y Lenguaje como de Pedagogía Terapéutica. En la etapa de Educación Primaria, la figura del maestro especialista en Audición y Lenguaje se intensificó, transformándose en un apoyo permanente dentro del aula de referencia.

Comunicación

- Hasta el momento de comenzar con el proceso de adquisición de lectura y escritura, la alumna utilizó un sistema de comunicación basado en el empleo de pictogramas desarrollados por El Portal Aragonés de Comunicación Aumentativa y Alternativa (ARASAAC).

- También se utilizaron, para mejorar el proceso de adquisición de aspectos metalingüísticos íntimamente relacionados con la lectura y la escritura, señalizadores fonéticos para efectuar una buena conversión fonemagrafema 